\title{
Associations of the Baltic Sea diet with cardiometabolic risk factors - a meta-analysis of three Finnish studies
}

\author{
Noora Kanerva $^{1,2 *}$, Niina E. Kaartinen ${ }^{1}$, Harri Rissanen ${ }^{3}$, Paul Knekt ${ }^{3}$, Johan G. Eriksson ${ }^{1,4,5,6}$, \\ Katri Sääksjärvi ${ }^{3}$, Jouko Sundvall ${ }^{1}$ and Satu Männistö ${ }^{1}$ \\ ${ }^{1}$ Department of Chronic Disease Prevention, National Institute for Health and Welfare, PO Box 30, \\ FI-O0271 Helsinki, Finland \\ ${ }^{2}$ Department of Public Health, University of Helsinki, Helsinki, Finland \\ ${ }^{3}$ Department of Health, Functional Capacity and Welfare, National Institute for Health and Welfare, Helsinki, Finland \\ ${ }^{4}$ Department of General Practice and Primary Health Care, University of Helsinki, Helsinki, Finland \\ ${ }^{5}$ Unit of General Practice, Helsinki University Central Hospital, Helsinki, Finland \\ ${ }^{6}$ Folkhälsan Research Center, Helsinki, Finland
}

(Submitted 10 September 2013 - Final revision received 1 April 2014 - Accepted 22 April 2014-First published online 27 May 2014)

\begin{abstract}
Dyslipidaemia, hypertension and low-grade inflammation increase the risk of CVD. In the present meta-analysis, we examined whether adherence to a healthy Nordic diet, also called the Baltic Sea diet, may associate with a lower risk of these cardiometabolic risk factors. In 2001-2007, three cross-sectional Finnish studies were conducted: the Dietary, Lifestyle and Genetic Determinants of Obesity and Metabolic Syndrome study ( $n$ 4776); Health 2000 Survey ( $n$ 5180); Helsinki Birth Cohort Study ( $n$ 1972). The following parameters were assessed in these three studies: blood pressure, total, HDL- and LDL-cholesterol, TAG and high-sensitivity C-reactive protein (hs-CRP); a validated FFQ was used to assess the participants' dietary intakes. The Baltic Sea Diet Score (BSDS) was developed based on the healthy Nordic diet. All studies assessed confounding variables, such as physical activity and BMI, based on standardised questionnaires and measurements. The random-effects meta-analysis provided summary estimates for OR and $95 \%$ CI by the BSDS quintiles. In the metaanalysis, the risk of elevated hs-CRP concentration was lower among men (OR 0.58, 95\% CI 0.43, 0.78) and women (OR 0.73, 95\% CI $0.58,0.91$ ) in the highest BSDS quintile than among those in the lowest BSDS quintile. In contrast, the risk of lowered HDL-cholesterol concentration was higher among women (OR 1.67, 95\% CI 1.12, 2.48) in the highest BSDS quintile than among those in the lowest BSDS quintile. However, no other associations were found. In conclusion, the associations between the adherence to the healthy Nordic diet and cardiometabolic risk factors are equivocal. Longitudinal studies are needed to further examine this hypothesis.
\end{abstract}

Key words: Baltic Sea diet: Nordic diet: C-reactive protein: Meta-analyses: Pooling

Elevated cholesterol and TAG concentrations, hypertension and low-grade inflammation increase the risk of chronic diseases, such as CVD which is a major cause of premature death in Western societies ${ }^{(1)}$. Any preventive measure taken against these risk factors may therefore contribute remarkably to public health. One of the most effective ways for improving cardiometabolic risk markers is through the modulation of the diet ${ }^{(2)}$. Researchers have extensively studied the effects of single foods and nutrients, e.g. fat, in relation to lipid metabolism; however, the interactions with other dietary components, such as carbohydrates, confound the interpretation of the evidence. Accordingly, research should focus on the overall diet. For such purposes, nutritionists have developed dietary scores that have been increasingly used in epidemiological studies ${ }^{(3)}$. Instead of using data-driven approaches to identify dietary patterns that exist among study participants, the dietary score method was employed, which is a hypothesis-oriented approach that predefines the diet and then assesses how well the participants adhere to it.

The most well-known dietary score, the Mediterranean Diet Score, is based on the traditional diet of the Mediterranean countries $^{(4)}$. Strong evidence exists that this diet improves metabolic risk factors; studies have reported normalisation of body weight and lipid concentrations as well as suppression

Abbreviations: BSDS, Baltic Sea Diet Score; DILGOM, Dietary, Lifestyle and Genetic Determinants of Obesity and Metabolic Syndrome; E\%, percentage of total energy intake; EI, energy intake; HBCS, Helsinki Birth Cohort Study; HDL-C, HDL-cholesterol; hs-CRP, high-sensitivity C-reactive protein; LDL-C, LDL-cholesterol.

*Corresponding author: N. Kanerva, email noora.kanerva@thl.fi 
of inflammation ${ }^{(5-7)}$. The diet also lowers the risk of CVDrelated mortality ${ }^{(8)}$. However, differences in food cultures, local resources and ecological aspects may hamper other populations, e.g. Scandinavians, from adopting the Mediterranean diet.

Many foods cultivated in the Nordic countries, for example, apples and berries, rye, rapeseed oil, salmon and dairy products, are considered to have health-enhancing features ${ }^{(9)}$. A Nordic diet compiled of these foods may, in theory, promote health ${ }^{(10)}$. Recently, controlled trials of individuals with hypercholesterolaemia or the metabolic syndrome have provided evidence that the healthy Nordic diet improves lipid profiles and reduces inflammation ${ }^{(11,12)}$. To measure the adherence to the healthy Nordic diet at a population level, a dietary score, called the Baltic Sea Diet Score (BSDS), was developed $^{(13)}$. The BSDS has been found to be associated with lower abdominal obesity in Finland ${ }^{(14)}$.

In the present study, we aimed to examine the associations between the adherence to the healthy Nordic diet and cardiometabolic risk factors (elevated total cholesterol, LDL-cholesterol (LDL-C), TAG and high-sensitivity C-reactive protein (hs-CRP) concentrations and hypertension, and lowered HDL-cholesterol (HDL-C) concentration) at the population level. We used the BSDS as an indicator of adherence to the healthy Nordic diet and analysed three Finnish health examination studies in a meta-analysis.

\section{Subjects and methods}

The present meta-analysis included three cross-sectional Finnish studies: the Dietary, Lifestyle and Genetic Determinants of Obesity and Metabolic Syndrome (DILGOM) study ( $n$ 5024); the Health 2000 Survey ( $n$ 8028); the clinical part of the Helsinki Birth Cohort Study (HBCS, $n$ 2003). The participants and the methods used in these studies have been described in detail elsewhere ${ }^{(15-18)}$. In brief, the DILGOM study was implemented in the framework of the National FINRISK 2007 Study that includes a representative sample of the Finnish population in five large study areas ${ }^{(16)}$. Participants who went through the health examination were invited to a more detailed health examination (=DILGOM) concerning obesity and the metabolic syndrome later during the same year ${ }^{(18)}$. The Health 2000 Survey aimed at determining the health and functional capability of $\geq 30$-year-old Finns, and collected information on the most important health disorders and the associated need for care, rehabilitation and aid ${ }^{(15)}$. The baseline survey was conducted during the year 2000-2001. A nationally representative sample was drawn from eighty health service districts throughout Finland. The HBCS investigated the effect of early growth on the later risk of non-communicable diseases ${ }^{(17)}$. The cohort members were born in 1934-1944 at Helsinki University Central Hospital as singletons. Using random number tables, a sample of 2902 participants from this cohort was derived and invited to a clinical examination conducted in 2001-2004.

The DILGOM study, the Health 2000 Survey and the HBCS were conducted according to the guidelines laid down in the Declaration of Helsinki, and all procedures involving human subjects were approved by the Ethics Committee of the Helsinki and Uusimaa Hospital District. Written informed consent was obtained from all subjects. Access to personal identification codes was restricted, and data were analysed without personal identification codes.

Dietary habits were assessed using the same semiquantitative $\mathrm{FFQ}^{(19)}$ and its two latest updated versions ${ }^{(20,21)}$ The updated versions did not differ substantially from each other. Participants recorded their food consumption during the previous year in nine frequency categories. The portion sizes in the FFQ were fixed for each food item and mixed dish in advance (e.g. by the glass or the slice). In the DILGOM study and the HBCS, participants completed the FFQ at the study site, where a trained study nurse reviewed the questionnaire. In the Health 2000 Survey, participants were asked to complete the FFQ at home after the health examination and send it back to the study centre. Researchers calculated the average daily food, nutrient and energy intakes (EI) using the national food composition database (Fineli ${ }^{\circledR}$ ) and in-house software ${ }^{(22)}$.

In all three studies, trained study nurses measured height, weight, waist circumference, and systolic and diastolic blood pressure using standard protocols ${ }^{(15,23,24)}$. All studies computed BMI $\left(\mathrm{kg} / \mathrm{m}^{2}\right)$. The study nurses drew blood for serum and plasma samples, which were stored at $-70^{\circ} \mathrm{C}$ until analysed. Research laboratories used automated clinical chemistry analysers and routine clinical chemistry methods to measure serum lipid fractions (total cholesterol, HDL-C and TAG concentrations) and hs-CRP concentration ${ }^{(25-27)}$ LDL-C concentrations were calculated using the Friedewald formula $^{(28)}$.

\section{Data handling}

The present meta-analysis consisted of participants who returned the FFQ form to the study centre (DILGOM study $n$ 4990, HBCS $n 2003$ and Health 2000 Survey $n$ 6373). The study was restricted to participants between 25 and 79 years of age, which led to the exclusion of 421 participants older than 79 years in the Health 2000 Survey. After exclusion of missing or incompletely filled FFQ (DILGOM study $n$ 74, HBCS $n 2$ and Health 2000 Survey $n$ 375), individuals with extremely low or high EI (DILGOM study ( $n$ 42) and HBCS ( $n$ 20): daily EI cut-offs corresponding to $0.5 \%$ at both ends of the daily EI distributions for men and women; Health 2000 Survey ( $n$ 18): daily EI values between $<2510$ and $>29288 \mathrm{~kJ} / \mathrm{d}(<600$ and $>7000 \mathrm{kcal} / \mathrm{d}))$, pregnant women (DILGOM study $n 27$ and Health 2000 Survey $n$ 34), and missing information of response variables (DILGOM study $n 71$, HBCS $n 9$ and Health 2000 Survey $n$ 345), the final meta-analysis data comprised 11928 participants (5494 men and 6434 women; DILGOM study $n$ 4776, HBCS $n 1972$ and Health 2000 Survey $n$ 5180).

The construction of the BSDS has been described in detail elsewhere $^{(13)}$. In short, the BSDS is based on the healthy Nordic diet ${ }^{(10)}$. The BSDS included nine dietary components: high intakes of Nordic fruits (apples and berries); Nordic vegetables (tomatoes, cucumbers, leafy vegetables, roots, 
cabbages and peas); Nordic cereals (rye, oats and barley); low-fat and fat-free milk; fish (salmon and freshwater fishes); high ratio of PUFA:(SFA + trans-fatty acids); low intakes of red and processed meat; total fat (as a percentage of total EI; E\%); moderate or low intake of alcohol (ethanol). In each study, the score components were divided using sexspecific quartiles of average daily intakes, and for each dietary component, except alcohol, points from 0 to 3 were assigned according to the predictable impact on health. For alcohol intake, participants received 1 point for moderate or lower intake $(\leq 20 \mathrm{~g} / \mathrm{d}$ of ethanol for men and $\leq 10 \mathrm{~g} / \mathrm{d}$ of ethanol for women) and 0 point if moderate intake was exceeded. The final score ranged from 0 to 25 .Higher scores indicate higher adherence to the healthy Nordic diet. The BSDS was divided into quintiles using study-specific quintile cut-offs, and used as the exposure variable.

We used international guidelines to define elevated or lowered values of cardiometabolic risk factors (later referred to as outcome variables). We transformed all the outcome variables to dummy variables, according to the cut-off values defined as follows. Elevated total cholesterol concentration was defined as $\geq 5.0 \mathrm{mmol} / 1$ or use of lipid-lowering medication ${ }^{(1)}$. Elevated LDL-C concentration was defined as $\geq 3.0 \mathrm{mmol} / \mathrm{l}$ or use of lipid-lowering medication ${ }^{(1)}$. We used harmonisation criteria of the metabolic syndrome for lowered HDL-C concentration $(<1.0 \mathrm{mmol} / \mathrm{l}$ for men and $<1.3 \mathrm{mmol} / \mathrm{l}$ for women $)^{(29)}$. According to the same criteria, we defined elevated TAG concentration as $\geq 1.7 \mathrm{mmol} / 1$, and elevated blood pressure as systolic blood pressure $\geq 130 \mathrm{mmHg}$ or diastolic blood pressure $\geq 85 \mathrm{mmHg}$ or use of blood pressure-lowering medication. Assessment of low-grade inflammation has no univocal recommendation; however, some evidence of higher risk for CVD exists at hs-CRP concentrations $\geq 3.0 \mathrm{mg} / \mathrm{l}^{(30)}$, which we used as the cut-off criteria. Furthermore, concentrations $>10 \mathrm{mg} / \mathrm{l}$ were excluded from hs-CRP analysis ( $n$ 415), since they were likely to indicate other than obesity-related inflammation.

All the three studies included questions on the participants' educational attainment, frequency of exercise (times per week) and smoking status. We used these questions to indicate socio-economic status, leisure-time physical activity and smoking status in the present meta-analysis. In the DILGOM study and the HBCS, medication use was obtained from the Social Insurance Institute's register for reimbursements of pharmaceutical expenses. In the Health 2000 Survey, medication use was self-reported.

\section{Statistical analyses}

Statistical analyses were performed using SAS version 9.2 (SAS Institute, Inc.) and $\mathrm{R}$ Statistical Software version 2.15.1 (31). Since the test for significance of the interaction between sex and the BSDS was $P<0.05$ for three (out of six) response variables, separate analyses were conducted for men and women. Descriptive data by the BSDS quintiles are reported as means with their standard errors or percentages. For all data, logistic regression was used to calculate OR and two-sided 95\% CI for each response variable by the BSDS quintiles in the three study cohorts. Potential confounding variables were first listed based on the literature. Second, we explored which of these variables were actual confounders in our data, i.e. independently associated with both the outcomes and exposure. This was done using linear regression analysis $^{(32)}$. For example, women's menopausal age was not associated with exposure; thus, it was not used as a confounding variable in the present study. The following confounding variables were included in modelling: age; leisure-time physical activity; smoking status; education; BMI; Na intake (only for hypertension). Furthermore, to take into account possible misreporting of EI, the ratio of reported EI:predicted BMR was calculated and the participants were classified as either underreporters (EI:BMR $\leq 1.14$ ) or plausible reporters (EI:BMR $>1 \cdot 14)^{(33-35)}(26 \%$ of the total participants). The final analyses were conducted with and without adjustment for under-reporting.

The random-effects meta-analysis was chosen as the analytic strategy. This two-stage process allows for betweenstudy diversity in study methods (e.g. different laboratories used) and heterogeneity in the associations between the outcome variables and exposure or covariates. In the metaanalysis, the pooled OR and its $95 \%$ CI for the mass of data were obtained by combining the study-specific log (OR) and weighing them by the inverse of their variance in a randomeffects model ${ }^{(36)}$. The $P$ value for test of trend was obtained from Wald's test of the pooled estimates using the median values of the BSDS quintiles as a continuous variable in the model. Homogeneity among the OR from the original studies was tested using the Q statistic.

In the logistic regression analysis, three main models were used. The first model was adjusted for age (years, continuous); the second model for age, leisure-time physical activity (categorical: $<1$ time/week, $1-3$ times/week and $\geq 4$ times/week), smoking status (categorical: non-smoker, former smoker and current smoker), education (years, continuous) and BMI ( $\mathrm{kg} / \mathrm{m}^{2}$, continuous). When hypertension was used as an outcome variable, $\mathrm{Na}$ intake ( $\mathrm{mg} / \mathrm{d}$, continuous) was also controlled in the second model. In the third model, the associations were examined by excluding the participants who were taking lipid-lowering medication (total cholesterol, LDL-C, HDL-C and TAG concentrations), blood pressurelowering medication (hypertension) or statin drugs (hs-CRP concentration), adjusting for the variables used in the second model. In interaction analyses, the associations between the outcome variables and the BSDS were examined in separate BMI and waist circumference categories.

\section{Results}

Participants with a higher adherence to the Baltic Sea diet were older, more physically active and more educated, less often smokers, had smaller waist circumference and were more likely to use lipid-lowering medication. Total cholesterol concentration decreased due to decreasing HDL-C concentration towards the highest BSDS quintile. No difference in the other lipid fractions was found between the BSDS quintiles, while the hs-CRP concentration tended to decrease 
towards the highest BSDS quintile. Of the participants included in the present meta-analysis, 83\% had elevated total serum cholesterol concentration or used lipid-lowering medication, $78 \%$ had elevated LDL-C concentration or used lipid-lowering medication, $18 \%$ of men and $29 \%$ of women had low HDL-C concentration, 37\% had elevated TAG concentration and $20 \%$ had elevated hs-CRP concentration.

The BSDS ranged from 1 to 25 points, and the quintile cut-offs were comparable between the studies (Table 1). The consumption of BSDS components considered healthy increased across the BSDS quintiles, whereas the consumption of components considered unhealthy decreased in all the three studies. In general, the DILGOM study tended to have the highest intakes of all score components, except the intakes of total fat and alcohol, which were highest in the HBCS.

The pooled risk of elevated hs-CRP concentration was lower by $42 \%$ in men (model 2; Table 2) and $27 \%$ in women (model 2; Table 3) in the highest BSDS quintile compared with the lowest. These associations remained after adjusting for under-reporters (data not shown). In contrast, the risk of lower HDL-C concentration was $40 \%$ higher among women (model 2), but not among men, in the highest BSDS quintile compared with the lowest. After adjusting for under-reporters, the association strengthened (data not shown). Among men, a positive trend between the higher BSDS and the risk of elevated LDL-C concentration was found (model 3; $P$ for trend=0.031), even though there was no statistically significant difference between the higher BSDS quintiles compared with the lowest. Adjusting for under-reporters did not affect this association (data not shown). We found no associations between the BSDS and other cardiometabolic risk factors. Furthermore, the associations between the BSDS and cardiometabolic risk factors were not modulated by the participants' BMI and waist circumference (data not shown).

When men using lipid-lowering medication were excluded from the analysis (17\% of the total participants), the OR in the fourth and fifth BSDS quintiles attenuated in relation to total cholesterol, LDL-C and TAG concentrations (model $2 v$. model 3; Table 2). Among women (19\% of the total participants), the OR did not change (model $2 v$. model 3; Table 3). In contrast, the OR for the risk of low HDL-C concentration strengthened among the two sexes, but the OR for men remained statistically non-significant. The OR for the risk of hypertension (excluding blood pressure-lowering medication users; $6 \%$ of the total participants) and elevated hs-CRP concentration (excluding statin users; $27 \%$ of the total participants) were similar among the two sexes. Adjusting for under-reporters did not affect the results (data not shown).

The positive trend across the BSDS quintiles between the higher BSDS and elevated LDL-C concentration disappeared after excluding men using lipid-lowering medication $(P=0 \cdot 40$, model 3; Table 2$)$. This result remained after adjusting for under-reporters (data not shown). Furthermore, the trend between the higher BSDS and elevated TAG concentration weakened among men ( $P=0 \cdot 34$, model 3; Table 2), but strengthened among women $(P=0 \cdot 09$, model 3; Table 3), when lipid-lowering medication users were excluded.
Furthermore, when statin users were excluded, the risk for elevated hs-CRP concentration attenuated $(P$ for trend $=0.05$ for men and $P$ for trend=0.17 for women); however, it became statistically significant again after adjusting for under-reporters in both sexes (data not shown).

To determine whether some of the BSDS components were driving the associations observed, we analysed the associations between individual BSDS components and elevated hs-CRP and lowered HDL-C concentrations. In men, higher intakes of fruits and berries, vegetables, cereals and low-fat milk decreased the risk of elevated hs-CRP concentration (Table 4). In women, higher intakes of fruits and berries, and cereals decreased this risk. In contrast, the risk of lower HDL-C concentration was increased by higher intakes of cereals and low-fat milk, and moderate or lower intake of alcohol, but decreased by the lower intake of total fat (as E\%) in women (data not shown).

\section{Discussion}

The present meta-analysis is among the first to examine the associations between the healthy Nordic diet, also called the Baltic Sea diet, and cardiometabolic risk factors. In the metaanalysis of three cross-sectional Finnish studies, we found that adherence to the healthy Nordic diet was associated with a lower risk of elevated hs-CRP concentration. This result was independent of the participants' age, leisure-time physical activity, education, smoking status and BMI, and remained significant after excluding statin users. In addition, among women, adherence to the healthy Nordic diet was associated with a higher risk of lower HDL-C concentration. This result strengthened after excluding lipid-lowering medication users. The risk of lower LDL-C concentration increased across the BSDS quintiles among men; however, the trend disappeared after excluding men using lipid-lowering medication. Adjusting for under-reporters did not affect these results.

Low-grade inflammation, for which elevated hs-CRP concentration is an important marker, is an independent risk factor for $\operatorname{CVD}^{(37,38)}$. Researchers have estimated that the assessment of hs-CRP concentration in individuals at intermediate risk for a cardiovascular event could help to prevent one additional event per 10 years for every 400-500 people screened ${ }^{(39)}$. Individuals at high risk for CVD could benefit from a hs-CRP concentration $<3.0 \mathrm{mmol} / 1$, regardless of their LDL-C concentration ${ }^{(37,38)}$. Adopting the Baltic Sea diet could therefore have a remarkable effect, since CVD is one of the leading causes of premature death in Finland ${ }^{(16)}$ However, trials studying the effects of healthy Nordic diet on subjects with hypercholesterolaemia and the metabolic syndrome did not show any decrease in hs-CRP concentration when compared with a control group ${ }^{(11,12)}$. Further research is thus needed to reveal whether there is a causal relationship between the Baltic Sea diet and hs-CRP concentration. In examining the individual BSDS components, we found that intakes of Nordic fruits and berries, and cereals were associated with a lower risk of elevated hs-CRP concentration. These foods are well known for their anti-inflammatory 
Table 1. Age- and sex-adjusted characteristics of the participants in the Dietary, Lifestyle and Genetic Determinants of Obesity and Metabolic Syndrome (DILGOM) study, Health 2000 Survey and Helsinki Birth Cohort Study (HBCS)

(Mean values with their standard errors or percentages)

\begin{tabular}{|c|c|c|c|c|c|c|c|c|c|c|c|c|c|c|c|c|c|c|}
\hline \multirow[b]{4}{*}{ Characteristics } & \multicolumn{6}{|c|}{ DILGOM study ( $n$ 4776) } & \multicolumn{6}{|c|}{ Health 2000 Survey ( $n$ 5180) } & \multicolumn{6}{|c|}{ HBCS ( $n$ 1972) } \\
\hline & \multicolumn{6}{|c|}{ BSDS quintiles } & \multicolumn{6}{|c|}{ BSDS quintiles } & \multicolumn{6}{|c|}{ BSDS quintiles } \\
\hline & \multicolumn{2}{|c|}{1} & \multicolumn{2}{|c|}{3} & \multicolumn{2}{|c|}{5} & \multicolumn{2}{|c|}{1} & \multicolumn{2}{|c|}{3} & \multicolumn{2}{|c|}{5} & \multicolumn{2}{|c|}{1} & \multicolumn{2}{|c|}{3} & \multicolumn{2}{|c|}{5} \\
\hline & Mean & SEM & Mean & SEM & Mean & SEM & Mean & SEM & Mean & SEM & Mean & SEM & Mean & SEM & Mean & SEM & Mean & SEM \\
\hline BSDS (range) & \multicolumn{2}{|c|}{$1-9$} & \multicolumn{2}{|c|}{$12-13$} & \multicolumn{2}{|c|}{$17-25$} & \multicolumn{2}{|c|}{$1-9$} & \multicolumn{2}{|c|}{$12-14$} & \multicolumn{2}{|c|}{$17-25$} & \multicolumn{2}{|c|}{$1-9$} & \multicolumn{2}{|c|}{$12-13$} & 17 & \\
\hline Age $^{\star}$ (years) & $47 \cdot 0$ & 0.4 & 53.0 & 0.4 & 58.5 & 0.4 & $46 \cdot 8$ & 0.4 & $50 \cdot 3$ & 0.4 & $55 \cdot 2$ & 0.4 & $61 \cdot 1$ & 0.2 & 61.5 & 0.2 & $61 \cdot 7$ & 0.1 \\
\hline Female† (\%) & 4 & & & & & & & & & & & & & & & & 5 & \\
\hline Energy under-reportersł (\%) & 3 & & & & & & & & & & & & & & & & 1 & \\
\hline Exercise $>4$ times/week (\%) & 2 & & & & & & & & & & & & & & & & 3 & \\
\hline Smokers (\%) & & & & & & & & & & & & & & & & & 1 & \\
\hline Years of education & $12 \cdot 2$ & 0.1 & $12 \cdot 6$ & 0.1 & $12 \cdot 9$ & 0.1 & $11 \cdot 0$ & 0.1 & $11 \cdot 6$ & 0.1 & $12 \cdot 1$ & 0.1 & 11.5 & 0.2 & $12 \cdot 2$ & 0.2 & $12 \cdot 7$ & 0.2 \\
\hline Lipid-lowering medication§ (\%) & & & & & & & & & & & & & & & & & 3 & \\
\hline BMI $\left(\mathrm{kg} / \mathrm{m}^{2}\right)$ & $27 \cdot 2$ & 0.2 & $26 \cdot 9$ & 0.2 & $26 \cdot 7$ & 0.2 & $26 \cdot 8$ & 0.1 & $27 \cdot 2$ & 0.2 & $26 \cdot 9$ & 0.2 & $27 \cdot 7$ & 0.2 & $27 \cdot 7$ & 0.3 & $27 \cdot 3$ & 0.2 \\
\hline WC $(\mathrm{cm})$ & 93.4 & 0.4 & 91.5 & 0.5 & 89.7 & 0.4 & $92 \cdot 6$ & 0.4 & $93 \cdot 3$ & 0.4 & 91.6 & 0.4 & $95 \cdot 9$ & 0.7 & $95 \cdot 6$ & 0.7 & 93.7 & 0.6 \\
\hline Body fat percentage & $30 \cdot 2$ & 0.3 & $30 \cdot 3$ & 0.3 & $30 \cdot 4$ & 0.3 & $27 \cdot 9$ & 0.3 & $27 \cdot 6$ & 0.3 & $27 \cdot 4$ & 0.3 & $30 \cdot 2$ & 0.4 & 28.9 & 0.5 & $28 \cdot 8$ & 0.4 \\
\hline Total cholesterol $(\mathrm{mmol} / \mathrm{l})$ & $5 \cdot 3$ & 0.03 & $5 \cdot 2$ & 0.03 & $5 \cdot 1$ & 0.03 & $6 \cdot 0$ & 0.03 & $6 \cdot 0$ & 0.03 & $5 \cdot 9$ & 0.03 & $6 \cdot 0$ & 0.06 & $6 \cdot 0$ & 0.06 & $5 \cdot 8$ & 0.05 \\
\hline TAG (mmol/)I & 1.3 & 0.03 & $1 \cdot 2$ & 0.03 & $1 \cdot 2$ & 0.02 & 1.6 & 0.03 & 1.6 & 0.03 & 1.6 & 0.03 & 1.6 & 0.05 & 1.6 & 0.06 & 1.4 & 0.05 \\
\hline HDL-cholesterol (mmol/l) & 1.5 & 0.01 & 1.5 & 0.01 & 1.4 & 0.01 & 1.4 & 0.01 & 1.3 & 0.01 & 1.3 & 0.01 & 1.6 & 0.02 & 1.6 & 0.02 & 1.6 & 0.02 \\
\hline LDL-cholesterol (mmol/l/) & $3 \cdot 2$ & 0.03 & $3 \cdot 2$ & 0.03 & $3 \cdot 2$ & 0.03 & $3 \cdot 8$ & 0.04 & 3.9 & 0.04 & 3.7 & 0.04 & 3.7 & 0.05 & 3.7 & 0.05 & 3.5 & 0.04 \\
\hline Systolic blood pressure (mmHg) & 135 & 1 & 133 & 1 & 135 & 1 & 134 & 1 & 134 & 1 & 134 & 1 & 146 & 1 & 145 & 1 & 144 & 1 \\
\hline Diastolic blood pressure $(\mathrm{mmHg})$ & 80 & 0.4 & 79 & 0.4 & 79 & 0.4 & 83 & $1 \cdot 0$ & 83 & $1 \cdot 0$ & 82 & $1 \cdot 0$ & 89 & 1.0 & 89 & $1 \cdot 0$ & 88 & 1.0 \\
\hline hs-CRP (mg/l) & $2 \cdot 8$ & 0.2 & $2 \cdot 1$ & 0.2 & $1 \cdot 7$ & 0.2 & $2 \cdot 5$ & 0.2 & $2 \cdot 2$ & 0.2 & 1.5 & 0.2 & 3.8 & 0.3 & 3.7 & 0.3 & 2.9 & 0.3 \\
\hline Fruits and berries (g/d) & 70 & 4 & 115 & 4 & 225 & 4 & 60 & 3 & 100 & 3 & 180 & 3 & 60 & 7 & 120 & 7 & 230 & 7 \\
\hline Vegetables $(\mathrm{g} / \mathrm{d})$ & 190 & 6 & 280 & 6 & 410 & 6 & 150 & 5 & 230 & 5 & 350 & 5 & 170 & 8 & 250 & 8 & 370 & 8 \\
\hline Cereals $(\mathrm{g} / \mathrm{d})$ & 56 & 1 & 78 & 1 & 113 & 1 & 42 & 1 & 61 & 1 & 88 & 1 & 33 & 2 & 55 & 2 & 84 & 2 \\
\hline Low-fat milk (g/d) & 190 & 10 & 270 & 10 & 390 & 9 & 180 & 8 & 260 & 8 & 340 & 8 & 160 & 13 & 205 & 13 & 280 & 12 \\
\hline Fish $(g / d)$ & 33 & 2 & 45 & 2 & 63 & 2 & 27 & 1 & 37 & 1 & 51 & 1 & 32 & 2 & 45 & 2 & 61 & 2 \\
\hline Red meat $(\mathrm{g} / \mathrm{d})$ & 180 & 2 & 140 & 2 & 90 & 2 & 165 & 2 & 130 & 2 & 90 & 2 & 162 & 4 & 115 & 4 & 69 & 3 \\
\hline Total fat (E\%) & $35 \cdot 3$ & 0.1 & 31.5 & 0.1 & $26 \cdot 8$ & 0.1 & $40 \cdot 2$ & 0.1 & $36 \cdot 1$ & 0.1 & $32 \cdot 3$ & 0.1 & $37 \cdot 1$ & 0.2 & $32 \cdot 7$ & 0.2 & $28 \cdot 6$ & 0.2 \\
\hline Fat ratio & 0.4 & $<0.1$ & 0.5 & $<0.1$ & 0.6 & $<0.1$ & 0.3 & $<0.1$ & 0.4 & $<0.1$ & 0.5 & $<0.1$ & 0.35 & $<0.1$ & 0.4 & $<0.1$ & 0.5 & $<0.1$ \\
\hline Alcohol (g/d) & 9.4 & 0.4 & $7 \cdot 6$ & 0.4 & $5 \cdot 6$ & 0.4 & 6.5 & 0.3 & 5.4 & 0.3 & $4 \cdot 3$ & 0.3 & 10.5 & 0.7 & $9 \cdot 2$ & 0.7 & 8.0 & 0.7 \\
\hline
\end{tabular}

BSDS, Baltic Sea Diet Score; WC, waist circumference; hs-CRP, high-sensitivity C-reactive protein; E\%, percentage of total energy intake.

*Adjusted only for sex.

‡Energy under-reporters were defined based on the Goldberg cut-off and the WHO definition as the ratio of reported energy intake:predicted BMR ${ }^{(32-34)}$.

$\S$ Lipid-lowering medication included in Anatomical Therapeutic Chemical Classification System code C10A. 
Table 2. Pooled risk of cardiometabolic risk factors by the Baltic Sea Diet Score (BSDS) in a Finnish meta-analysis of men

(Pooled odds ratios and $95 \%$ confidence intervals)

\begin{tabular}{|c|c|c|c|c|c|c|c|c|c|c|c|}
\hline \multirow[b]{3}{*}{ Models } & \multicolumn{11}{|c|}{ BSDS quintiles } \\
\hline & \multirow{2}{*}{$\begin{array}{l}1 \text { ( } n \text { 1146) } \\
\text { Reference }\end{array}$} & \multicolumn{2}{|c|}{$2(n 1119)$} & \multicolumn{2}{|c|}{$3(n$ 1022) } & \multicolumn{2}{|c|}{$4(n 1127)$} & \multicolumn{2}{|c|}{$5(n 1080)$} & \multirow[b]{2}{*}{$P^{*}$} & \multirow[b]{2}{*}{$P \dagger$} \\
\hline & & OR & $95 \% \mathrm{Cl}$ & OR & $95 \% \mathrm{Cl}$ & OR & $95 \% \mathrm{Cl}$ & OR & $95 \% \mathrm{Cl}$ & & \\
\hline Cholesterol concentration $\geq 5.0 \mathrm{mmol} / /$ or lipid-lowering medication $(n)$ & 905 & & 889 & & 828 & & 892 & & 902 & & \\
\hline Model $1 \ddagger$ & 1.00 & 0.85 & $0.65,1.12$ & 0.94 & $0.74,1.17$ & 0.88 & $0.66,1.16$ & 0.95 & $0.68,1.34$ & 0.68 & 0.23 \\
\hline Model $2 \S$ & 1.00 & 0.87 & $0.62,1.22$ & 0.98 & $0.77,1 \cdot 24$ & 0.94 & $0.65,1.37$ & 1.04 & $0.72,1.50$ & 0.80 & $0 \cdot 18$ \\
\hline Without medication users $(n)$ & 666 & & 617 & & 544 & & 543 & & 511 & & \\
\hline Model 3\| & 1.00 & 0.80 & $0.57,1.12$ & 0.92 & $0.72,1.18$ & 0.80 & $0.63,1.02$ & 0.87 & $0.65,1.17$ & 0.25 & 0.42 \\
\hline LDL-C concentration $\geq 3 \mathrm{mmol} / \mathrm{l}$ or lipid-lowering medication $(n)$ & 878 & & 893 & & 840 & & 923 & & 909 & & \\
\hline Model 1 & 1.00 & 1.05 & $0.86,1.30$ & 1.21 & $0.97,1.52$ & 1.17 & $0.94,1.45$ & $1 \cdot 15$ & $0.92,1.44$ & $0 \cdot 10$ & 0.71 \\
\hline Model 2 & 1.00 & 1.07 & $0.86,1.32$ & 1.24 & $0.97,1.58$ & 1.21 & $0.97,1.52$ & 1.23 & $0.97,1.56$ & 0.031 & 0.61 \\
\hline Without medication users $(n)$ & 645 & & 626 & & 559 & & 579 & & 516 & & \\
\hline Model 3 & 1.00 & 1.05 & $0.83,1.31$ & $1 \cdot 16$ & $0.91,1.48$ & $1 \cdot 18$ & $0.83,1.67$ & 1.07 & $0.81,1.40$ & 0.40 & 0.20 \\
\hline HDL-C concentration $<1.0 \mathrm{mmol} / \mathrm{l}(n)$ & 210 & & 218 & & 173 & & 195 & & 204 & & \\
\hline Model 1 & 1.00 & 0.99 & $0.72,1.35$ & 0.92 & $0.73,1.16$ & 1.01 & $0.74,1.38$ & 0.99 & $0.69,1.43$ & 0.95 & 0.17 \\
\hline Model 2 & 1.00 & 1.00 & $0.69,1.44$ & 0.93 & $0.73,1.18$ & 1.08 & $0.81,1.46$ & 1.14 & $0.77,1.69$ & 0.41 & 0.19 \\
\hline Without medication users $(n)$ & 171 & & 181 & & 129 & & 142 & & 131 & & \\
\hline Model 3 & 1.00 & $1 \cdot 15$ & $0.89,1.48$ & 0.92 & $0.70,1.22$ & $1 \cdot 15$ & $0.86,1.54$ & $1 \cdot 21$ & $0.90,1.61$ & 0.43 & 0.30 \\
\hline TAG concentration $\geq 1.7 \mathrm{mmol} / \mathrm{l}(n)$ & 465 & & 489 & & 444 & & 491 & & 532 & & \\
\hline Model 1 & 1.00 & 1.06 & $0.89,1.26$ & 1.00 & $0.84,1.19$ & 0.97 & $0.82,1.16$ & $1 \cdot 14$ & $0.94,1.37$ & 0.34 & 0.44 \\
\hline Model 2 & 1.00 & 1.05 & $0.87,1.27$ & 1.02 & $0.85,1.23$ & 1.04 & $0.87,1.26$ & 1.29 & $0.98,1.71$ & $0 \cdot 11$ & 0.15 \\
\hline Without medication users $(n)$ & 285 & & 289 & & 218 & & 203 & & 203 & & \\
\hline Model 3 & 1.00 & 1.03 & $0.83,1.29$ & 0.89 & $0.70,1 \cdot 31$ & 0.85 & $0.67,1.09$ & 0.96 & $0.75,1.24$ & 0.34 & 0.88 \\
\hline Hypertension $9(n)$ & 804 & & 797 & & 720 & & 832 & & 807 & & \\
\hline Model 1 & 1.00 & 0.96 & $0 \cdot 71,1 \cdot 30$ & 0.85 & $0.70,1.03$ & 0.97 & $0.75,1.24$ & 0.90 & $0.67,1.20$ & 0.54 & 0.07 \\
\hline Model 2 & 1.00 & 0.97 & $0.67,1.37$ & 0.85 & $0.69,1.05$ & 1.00 & $0.72,1.38$ & 0.92 & $0 \cdot 73,1 \cdot 16$ & 0.67 & 0.14 \\
\hline Without medication users $(n)$ & 591 & & 548 & & 483 & & 541 & & 473 & & \\
\hline Model 3 & 1.00 & 0.96 & $0.66,1.39$ & 0.84 & $0.68,1.04$ & 0.99 & $0.72,1.34$ & 0.91 & $0.68,1.22$ & 0.65 & 0.11 \\
\hline hs-CRP concentration $\geq 3.0 \mathrm{mg} / \mathrm{l}(n)$ & 243 & & 211 & & 190 & & 208 & & 154 & & \\
\hline Model 1 & 1.00 & 0.78 & $0.63,0.98$ & 0.74 & $0.60,0.92$ & 0.70 & $0.56,0.87$ & 0.46 & $0.32,0.67$ & $<0.001$ & $0 \cdot 10$ \\
\hline Model 2 & 1.00 & 0.83 & $0.66,1.04$ & 0.81 & $0.64,1.02$ & 0.83 & $0.62,1.10$ & 0.58 & $0.43,0.78$ & 0.004 & 0.15 \\
\hline Without statin users $(n)$ & 159 & & 137 & & 106 & & 104 & & 81 & & \\
\hline Model 3 & 1.00 & 0.85 & $0 \cdot 64,1 \cdot 12$ & 0.76 & $0.57,1.01$ & 0.68 & $0.50,0.91$ & 0.59 & $0.36,0.97$ & 0.05 & 0.53 \\
\hline
\end{tabular}

LDL-C, LDL-cholesterol; HDL-C, HDL-cholesterol; hs-CRP, high-sensitivity C-reactive protein.

* Two-sided $P$ value (Wald's test) for linear trend across the BSDS quintiles was tested, using the median values of the BSDS quintiles as continuous variables.

*Two-sided $P$ value (Wald's test) for linear trend across the
$\dagger$ Two-sided $P$ value for heterogeneity between the studies.

$\ddagger$ Adjusted for age.

$\S$ Adjusted for age, physical activity, smoking status, education and BMI.

$\|$ Model 3 is model 2 excluding lipid-lowering medication (total cholesterol, LDL-C, HDL-C and TAG concentrations), blood pressure-lowering medication (hypertension) or statin (hs-CRP concentration) users.

q Hypertension was defined as systolic blood pressure $\geq 130 \mathrm{mmHg}$ or diastolic blood pressure $\geq 85 \mathrm{mmHg}$ or use of blood pressure-lowering medication. For hypertension, model 2 was also adjusted for Na intake (mg/d, continuous). 
Table 3. Pooled risk of cardiometabolic risk factors by the Baltic Sea Diet Score (BSDS) in a Finnish meta-analysis of women

(Pooled odds ratios and $95 \%$ confidence intervals)

\begin{tabular}{|c|c|c|c|c|c|c|c|c|c|c|c|}
\hline \multirow[b]{3}{*}{ Models } & \multicolumn{11}{|c|}{ BSDS quintiles } \\
\hline & \multirow{2}{*}{$\begin{array}{l}1(n \text { 1260) } \\
\text { Reference }\end{array}$} & \multicolumn{2}{|c|}{$2(n 1165)$} & \multicolumn{2}{|c|}{$3(n 1110)$} & \multicolumn{2}{|c|}{$4(n 1520)$} & \multicolumn{2}{|c|}{$5(n$ 1379) } & \multirow[b]{2}{*}{$P^{*}$} & \multirow[b]{2}{*}{$P \dagger$} \\
\hline & & OR & $95 \% \mathrm{Cl}$ & OR & $95 \% \mathrm{Cl}$ & OR & $95 \% \mathrm{Cl}$ & OR & $95 \% \mathrm{Cl}$ & & \\
\hline Cholesterol concentration $\geq 5.0 \mathrm{mmol} / /$ or lipid-lowering medication $(n)$ & 920 & & 832 & & 852 & & 1167 & & 1149 & & \\
\hline Model $1 \ddagger$ & 1.00 & 0.85 & $0.70,1.05$ & 0.91 & $0.74,1.12$ & 0.84 & $0.69,1.02$ & 0.94 & $0.75,1 \cdot 19$ & 0.45 & 0.67 \\
\hline Model $2 \S$ & 1.00 & 0.87 & $0.71,10 \cdot 7$ & 0.93 & $0.75,1.16$ & 0.86 & $0.70,1.06$ & 1.01 & $0.80,1.26$ & 0.77 & 0.80 \\
\hline Without medication users $(n)$ & 752 & & 655 & & 653 & & 851 & & 797 & & \\
\hline Model 3\| & 1.00 & 0.89 & $0 \cdot 71,1 \cdot 10$ & 0.92 & $0.74,1 \cdot 15$ & 0.84 & $0.68,1.03$ & 0.93 & $0 \cdot 70,1 \cdot 23$ & 0.36 & 0.48 \\
\hline LDL-C concentration $\geq 3 \mathrm{mmol} / \mathrm{l}$ or lipid-lowering medication $(n)$ & 903 & & 804 & & 835 & & 1167 & & 1122 & & \\
\hline Model 1 & 1.00 & 0.84 & $0.69,1.03$ & 0.94 & $0.77,1.15$ & 0.95 & $0.79,1 \cdot 16$ & 0.91 & $0.62,1.33$ & 0.84 & 0.09 \\
\hline Model 2 & 1.00 & 0.88 & $0.71,1.07$ & 0.98 & $0.80,1.22$ & 0.99 & $0.81,1 \cdot 21$ & 1.04 & $0.75,1.44$ & 0.51 & 0.23 \\
\hline Without medication users $(n)$ & 736 & & 628 & & 641 & & 853 & & 775 & & \\
\hline Model 3 & 1.00 & 0.89 & $0.71,1.09$ & 0.99 & $0.80,1 \cdot 24$ & 0.97 & $0 \cdot 79,1 \cdot 20$ & 0.97 & $0.68,1.40$ & 0.94 & 0.14 \\
\hline HDL-C concentration $<1.3 \mathrm{mmol} / /(n)$ & 342 & & 331 & & 301 & & 451 & & 410 & & \\
\hline Model 1 & 1.00 & $1 \cdot 13$ & $0.94,1.36$ & 1.03 & $0.80,1.33$ & 1.15 & $0.97,1.36$ & $1 \cdot 15$ & $0.94,1.41$ & $0 \cdot 11$ & 0.38 \\
\hline Model 2 & 1.00 & 1.22 & $1.00,1.48$ & $1 \cdot 11$ & $0.91,1.36$ & 1.29 & $1.07,1.55$ & 1.40 & $1.07,1.83$ & 0.006 & 0.25 \\
\hline Without medication users $(n)$ & 300 & & 278 & & 255 & & 356 & & 328 & & \\
\hline Model 3 & 1.00 & $1 \cdot 20$ & $0.97,1.48$ & $1 \cdot 16$ & $0.93,1.44$ & $1 \cdot 29$ & $1 \cdot 06,1 \cdot 58$ & $1 \cdot 67$ & $1 \cdot 12,2 \cdot 48$ & 0.007 & 0.14 \\
\hline TAG concentration $\geq 1.7 \mathrm{mmol} / \mathrm{l}(n)$ & 323 & & 326 & & 335 & & 498 & & 428 & & \\
\hline Model 1 & 1.00 & 0.96 & $0.79,1.17$ & 0.96 & $0.79,1.17$ & 1.03 & $0.86,1.23$ & 0.90 & $0.74,1.08$ & 0.49 & 0.72 \\
\hline Model 2 & 1.00 & 1.01 & $0.83,1.24$ & 1.06 & $0.81,1.38$ & 1.14 & $0.89,1.47$ & 1.08 & $0.89,1.32$ & 0.20 & 0.89 \\
\hline Without medication users $(n)$ & 200 & & 177 & & 165 & & 225 & & 170 & & \\
\hline Model 3 & 1.00 & 1.04 & $0.81,1 \cdot 34$ & 0.99 & $0.77,1 \cdot 28$ & 1.05 & $0 \cdot 75,1.47$ & 0.68 & $0.43,1.06$ & 0.08 & 0.54 \\
\hline Hypertensionๆ $(n)$ & 649 & & 629 & & 641 & & 912 & & 919 & & \\
\hline Model 1 & 1.00 & 0.95 & $0.79,1 \cdot 14$ & 0.98 & $0.81,1.18$ & 1.05 & $0.80,1.39$ & 1.04 & $0.87,1.25$ & 0.44 & 0.97 \\
\hline Model 2 & 1.00 & 0.95 & $0.78,1.15$ & 0.96 & $0.79,1.17$ & 1.05 & $0.82,1.33$ & 1.07 & $0.88,1.31$ & 0.37 & 0.97 \\
\hline Without medication users $(n)$ & 512 & & 491 & & 489 & & 667 & & 641 & & \\
\hline Model 3 & 1.00 & 0.97 & $0 \cdot 80,1 \cdot 18$ & 0.99 & $0 \cdot 81,1 \cdot 20$ & 1.04 & $0 \cdot 82,1 \cdot 32$ & 1.08 & $0 \cdot 89,1 \cdot 31$ & 0.39 & 0.79 \\
\hline hs-CRP concentration $\geq 3.0 \mathrm{mg} / \mathrm{l}(\mathrm{n})$ & 300 & & 269 & & 240 & & 306 & & 251 & & \\
\hline Model 1 & 1.00 & 0.93 & $0.76,1.12$ & 0.84 & $0.69,1.03$ & 0.76 & $0.63,0.92$ & 0.65 & $0.53,0.79$ & $<0.001$ & 0.48 \\
\hline Model 2 & 1.00 & 0.98 & $0.79,1.21$ & 0.91 & $0.73,1.13$ & 0.81 & $0.66,1.00$ & 0.73 & $0.58,0.91$ & 0.001 & 0.83 \\
\hline Without statin users $(n)$ & 208 & & 171 & & 158 & & 201 & & 140 & & \\
\hline Model 3 & 1.00 & 0.94 & $0.73,1.21$ & 0.93 & $0.72,1.20$ & 0.92 & $0.69,1.22$ & 0.69 & $0.53,0.91$ & 0.17 & 0.92 \\
\hline
\end{tabular}


properties, which may be the mechanism behind the lower risk $^{(40-42)}$.

In contrast to the findings on hs-CRP, the present results showed that adherence to the Baltic Sea diet was associated with a higher risk of lower HDL-C concentration, especially in women. A moderate or low intake of alcohol seemed to be the most important factor driving this association. Moderate alcohol consumption has long been known to increase HDL-C concentrations $^{(43)}$. High intakes of cereals and low-fat milk were associated with lower HDL-C concentration, which may relate to the high content of added sugars in some frequently used products (confectioneries and yogurts); however, the evidence is inconsistent ${ }^{(44)}$. A low-fat diet may decrease HDL-C concentration; however, this hypothesis also remains speculative ${ }^{(45-47)}$. In the present study, participants in the highest BSDS quintile had a mean total fat intake of $29.5 \mathrm{E} \%$, which fits the Nordic nutrition recommendations ${ }^{(48)}$. Changes in HDL-C concentration observed in Nordic diet trials with comparable total fat estimates (E\%) in the intervention group have also produced inconsistent results ${ }^{(11,12)}$.

We hypothesised that there would be inverse associations between the BSDS and the risk of elevated total cholesterol, LDL-C and TAG concentrations. In contrast, the risk of high LDL-C concentration increased across the BSDS quintiles, and no difference in the risk of elevated total cholesterol and TAG concentrations between the BSDS quintiles appeared. This finding may partly relate to medication use. Since diet plays a crucial role alongside medication in the treatment of dyslipidaemia ${ }^{(49)}$, it is highly likely that health professionals have advised individuals with lipid-lowering medication to change their diet. In cross-sectional settings, this may evoke a positive association between a healthy diet and a cardiometabolic risk factor. Regarding total cholesterol, LDL-C and TAG, our sensitivity analysis supports this hypothesis, but then again, the analysis does not support this hypothesis for HDL-C. Thus, we cannot rule out the possibility that the diet may actually increase the risks. However, our evidence suggests that medication users are following a healthier diet that may have resulted from receiving dietary counselling. Furthermore, there may be some factors that we were not able to take into account in the present meta-analysis, for example genetic variation in apoE that may confound or modulate the risks of adverse cholesterol concentrations.

We found no association between the BSDS and hypertension. The use of blood pressure-lowering medication did not seem to modulate the result. However, this is, in fact, a positive finding since we earlier observed that adherence to the diet was associated with higher Na intake, the most important

Table 4. Elevated high-sensitivity C-reactive protein (hs-CRP) concentrations by Baltic Sea Diet Score (BSDS) components in a Finnish meta-analysis* ${ }^{*}$

(Pooled odds ratios and 95\% confidence intervals)

\begin{tabular}{|c|c|c|c|c|c|c|c|c|c|}
\hline \multirow[b]{3}{*}{ BSDS components $\ddagger$} & \multicolumn{7}{|c|}{ BSDS component quartile } & \multirow[b]{3}{*}{$P \S$} & \multirow[b]{3}{*}{$P \|$} \\
\hline & \multirow{2}{*}{$\begin{array}{c}1 \\
1 \text { (Reference) }\end{array}$} & \multicolumn{2}{|r|}{2} & \multicolumn{2}{|r|}{3} & \multicolumn{2}{|r|}{4} & & \\
\hline & & OR & $95 \% \mathrm{Cl}$ & OR & $95 \% \mathrm{Cl}$ & OR & $95 \% \mathrm{Cl}$ & & \\
\hline \multicolumn{10}{|l|}{ Men ( $n$ 5494) } \\
\hline Fruits and berries & 1.00 & 0.89 & $0.73,1.09$ & 0.74 & $0.60,0.91$ & 0.71 & $0.56,0.89$ & 0.019 & 0.28 \\
\hline Vegetables & 1.00 & 0.86 & $0.70,1.06$ & 0.82 & $0.66,1.01$ & 0.73 & $0.59,0.89$ & 0.029 & 0.10 \\
\hline Cereals & 1.00 & 0.90 & $0.73,1.10$ & 0.84 & $0.68,1.03$ & $0 \cdot 81$ & $0.65,1.00$ & 0.031 & 0.51 \\
\hline Low-fat milkq & 1.00 & 0.89 & $0.73,1.09$ & 0.81 & $0.66,0.99$ & 0.80 & $0.65,0.98$ & 0.027 & 0.78 \\
\hline Fish & 1.00 & 0.82 & $0.66,1.01$ & 0.85 & $0.70,1.06$ & 0.89 & $0.72,1.10$ & 0.78 & 0.78 \\
\hline Meat products & 1.00 & 1.07 & $0.86,1.32$ & 1.25 & $0.97,1.62$ & 1.00 & $0.80,1.23$ & 0.66 & 0.83 \\
\hline Fat ratio** & 1.00 & 0.97 & $0.69,1.37$ & $1 \cdot 10$ & $0.89,1.35$ & 0.92 & $0.66,1.28$ & 0.20 & 0.35 \\
\hline Total fat (E\%) & 1.00 & 1.06 & $0.76,1.49$ & 0.96 & $0.78,1.19$ & $1 \cdot 18$ & $0.96,1.45$ & 0.36 & 0.74 \\
\hline Alcohol & $1.00 \mathrm{tt}$ & 1.07 & $0.86,1.33$ & & & & & 0.85 & 0.89 \\
\hline \multicolumn{10}{|l|}{ Women ( $n$ 6434) } \\
\hline Fruits and berries & 1.00 & 0.93 & $0.77,1.11$ & 0.78 & $0.65,0.95$ & 0.80 & $0.65,0.96$ & 0.07 & 0.76 \\
\hline Vegetables & 1.00 & 1.11 & $0.73,1.68$ & 0.96 & $0.80,1.16$ & 1.01 & $0.74,1.38$ & 0.32 & 0.32 \\
\hline Cereals & 1.00 & 1.00 & $0.83,1.21$ & 0.90 & $0.74,1.09$ & 0.81 & $0.66,0.98$ & 0.028 & 0.77 \\
\hline Low-fat milkq & 1.00 & 0.92 & $0.76,1.11$ & 0.95 & $0.79,1.14$ & 0.99 & $0.83,1.20$ & 0.59 & 0.99 \\
\hline Fish & 1.00 & 1.04 & $0.85,1.27$ & 0.87 & $0.68,1.11$ & 0.90 & $0.70,1.56$ & 0.44 & 0.09 \\
\hline Meat products & 1.00 & 1.05 & $0.86,1.27$ & $1 \cdot 14$ & $0.94,1.39$ & $1 \cdot 15$ & $0.94,1.39$ & 0.11 & 0.77 \\
\hline Fat ratio** & 1.00 & 1.07 & $0.78,1.45$ & $1 \cdot 16$ & $0.81,1.66$ & 1.02 & $0.74,1.40$ & 0.11 & 0.12 \\
\hline Total fat (E\%) & 1.00 & 1.19 & $0.99,1.44$ & $1 \cdot 16$ & $0.96,1.40$ & $1 \cdot 14$ & $0.94,1.38$ & 0.98 & 0.34 \\
\hline Alcohol & $1.00 t t$ & 1.03 & $0.77,1.36$ & & & & & 0.99 & 0.25 \\
\hline
\end{tabular}

$\mathrm{E} \%$, percentage of total energy intake.

${ }^{*}$ Model was adjusted for age, physical activity, smoking status and BMI.

$\dagger$ Elevated hs-CRP concentration defined as $\geq 3.0 \mathrm{mmol} / \mathrm{l}$.

$\ddagger$ Study- and sex-specific cut-off quartiles were used. Participants in the higher quartiles of the presumably beneficial effect were assigned higher points than those in the lowest quartiles. In contrast, participants in the lower quartiles of the presumably detrimental effects were assigned higher points than those in the highest quartiles.

$\S$ Two-sided $P$ value was obtained from Wald's test, using intake of each component as a continuous variable in the model.

$\|$ Two-sided $P$ value for heterogeneity between the studies.

I Fat-free milk and milk with $<2 \%$ fat.

${ }^{* \star}$ Ratio of PUFA:(SFA + trans-fatty acids).

$\dagger †$ Men who consumed $\leq 20 \mathrm{~g} / \mathrm{d}$ of alcohol and women who consumed $\leq 10 \mathrm{~g} / \mathrm{d}$ of alcohol received 1 point, otherwise a 0 point was given. Thus, for alcohol, point 0 was assigned to the reference group and point 1 assigned to the adherence to the Baltic Sea diet. 
dietary risk factor for hypertension ${ }^{(13)}$. In other words, the result suggests that despite slightly higher $\mathrm{Na}$ intake, there is no evidence that participants adhering to the diet had a higher risk of hypertension. Furthermore, the BSDS does not include any specific measure for $\mathrm{Na}$ intake, which may hamper the detection of its association with hypertension.

The strengths of the present meta-analysis were its high power and the population-based approach in the DILGOM study and the Health 2000 Survey. Validated and standardised questionnaires and measurements, as well as robust and careful statistical testing, were the other strengths of the present study. All the three studies used the same FFQ, which is considered to be a standard method for assessing long-term dietary intakes in large epidemiological studies ${ }^{(50)}$. The FFQ has been validated for food and nutrient intakes and reproducibility against food diaries ${ }^{(19-21)}$. Some studies have shown that dietary scores provide a valid estimate of diet quality when they are based on the FFQ method ${ }^{(51)}$. We used a cut-off of $\geq 3.0 \mathrm{mg} / \mathrm{l}$ for defining elevated hs-CRP concentration $^{(30)}$. In preliminary analyses, we tested several cut-offs (e.g. $>1.0,>2.0$ and $>3.0 \mathrm{mg} / \mathrm{l}$ ) that produced similar results, indicating that the association is not dependent on the cut-off chosen (data not shown). The statistically significant trend test also supports this conclusion.

The present study also has some limitations. As discussed earlier, the cross-sectional design limits the conclusions that can be drawn. Overestimation of healthy and underestimation of unhealthy food consumption could have led to some misclassifications in the BSDS quintiles or weakened the associations observed $^{(52)}$. However, we aimed to control this by excluding participants with extreme EI, and adjusting the analyses for energy under-reporting. A recent study focusing on methodological aspects in relation to HDL-C concentration analyses in Finland showed that various direct HDL-C assays may have led to unexpected misclassifications of some participants ${ }^{(53)}$. This may have affected the HDL-C association observed to some extent. Furthermore, HDL-C subfraction (HDL2- and HDL3-cholesterol) concentrations were not measured. Even though the role of these subfractions in the aetiology of atherosclerosis or thrombosis is not well understood, the majority of published reports have linked the cardioprotective properties of HDL-C with HDL2 ${ }^{(54,55)}$. Analysing the associations between the BSDS and these subfractions would have eased the interpretation of the present results.

The dietary score method has its strengths and limitations. Dietary scores have the advantage to take into account the cumulative effects and interactions between several foods and nutrients. The score cut-off method may have led to participants in the highest quintile having different dietary profiles. For example, within the third BSDS quintile, some participants may have received only 1 point by consuming Nordic cereals while some participants obtained 3 points. The cut-off for the highest BSDS quintile was 17 points in each study. This high cut-off requires higher consumption than the population median for most of the components; in case of unhealthy components, this requires lower consumption than the population median. Furthermore, the results from the DILGOM study showed that participants within the highest quintile (cut-off 17 points) had similar nutrient intake profiles, for example similar intakes of fibre, vitamins $\mathrm{C}$ and $\mathrm{D}$, total fat and alcohol $^{(13)}$. In addition, the consumption of BSDS components was fairly similar between the studies in the present meta-analysis.

In conclusion, high adherence to the Baltic Sea diet may lower the risk of low-grade inflammation among Finns, which may decrease the risk of chronic diseases among the average population. Nevertheless, high adherence to the diet may set women at a higher risk of lower HDL-C concentration. The health consequences of these finding are controversial, since, for example, the cardioprotective properties of HDL-C subfractions ${ }^{(54,55)}$, the causal relationship between HDL-C concentration and CVD risk ${ }^{(56)}$ and the reliability of HDL-C assays remain uncertain ${ }^{(53)}$. Further longitudinal studies are needed to verify the associations between the Baltic Sea diet and the risk of CVD.

\section{Acknowledgements}

The present meta-analysis was supported by the Finnish Foundation for Cardiovascular Research (to N. K.). The DILGOM study was funded by the Academy of Finland (to S. M., grant no. 136895 and 263836). The HBCS was supported by grants from the Academy of Finland (to J. G. E., grant no. 120386 and 125876), the Finnish Diabetes Research Society, Folkhälsan Research Foundation, Novo Nordisk Foundation, Finska Läkaresällskapet, Liv and Hälsa, Samfundet Folkhälsan, Finska Läkaresällskapet and the Signe and Ane Gyllenberg Foundation.

The authors' responsibilities were as follows: S. M. and J. G. E. participated in the design of the DILGOM Study; P. K. participated in the conception of the Health 2000 Survey; J. G. E. participated in the design of the HBCS; P. K. participated in the development of the statistical programs utilised; J. S. conducted laboratory analysis of the DILGOM Study and the Health 2000 Survey; N. K., N. E. K. and H. R. handled and analysed the data; N. K. wrote the manuscript and had primary responsibility for the final content; K. S. critically revised the manuscript.

The authors had no financial conflicts of interest relevant to this article.

\section{References}

1. Fifth Joint Task Force of the European Society of Cardiology, European Association of Echocardiography, European Association of Percutaneous Cardiovascular Interventions, et al. (2012) European Guidelines on cardiovascular disease prevention in clinical practice (version 2012). The Fifth Joint Task Force of the European Society of Cardiology and Other Societies on Cardiovascular Disease Prevention in Clinical Practice (constituted by representatives of nine societies and by invited experts). Eur J Prev Cardiol 19, 585-667.

2. Mente A, de Köning L, Shannon HS, et al. (2009) A systematic review of the evidence supporting a causal link between dietary factors and coronary heart disease. Arch Intern Med 169, 659-669. 
3. Wirt A \& Collins CE (2009) Diet quality - what is it and does it matter? Public Health Nutr 12, 2473-2492.

4. Trichopoulou A, Kouris-Blazos A, Wahlqvist ML, et al. (1995) Diet and overall survival in elderly people. BMJ 311, 1457-1460.

5. Esposito K, Marfella R, Ciotola M, et al. (2004) Effect of a Mediterranean-style diet on endothelial dysfunction and markers of vascular inflammation in the metabolic syndrome: a randomized trial. JAMA 292, 1440-1446.

6. Babio N, Bulló M \& Salas-Salvadó J (2009) Mediterranean diet and metabolic syndrome: the evidence. Public Health Nutr 12, 1607-1617.

7. Kastorini CM, Milionis HJ, Esposito K, et al. (2011) The effect of Mediterranean diet on metabolic syndrome and its components: a meta-analysis of 50 studies and 534,906 individuals. J Am Coll Cardiol 57, 1299-1313.

8. Sofi F, Abbate R, Gensini GF, et al. (2010) Accruing evidence on benefits of adherence to the Mediterranean diet on health: an updated systematic review and meta-analysis. Am J Clin Nutr 92, 1189-1196.

9. Bere E \& Brug J (2009) Towards health-promoting and environmentally friendly regional diets - a Nordic example. Public Health Nutr 12, 91-96.

10. Adamsson V, Reumark A, Cederholm T, et al. (2012) What is a healthy Nordic diet? Foods and nutrients in the NORDIET study. Food Nutr Res 56 (Epublication ahead of print version 27 June 2012).

11. Adamsson V, Reumark A, Fredriksson IB, et al. (2011) Effects of a healthy Nordic diet on cardiovascular risk factors in hypercholesterolaemic subjects: a randomized controlled trial (NORDIET). J Intern Med 269, 150-159.

12. Uusitupa M, Hermansen K, Savolainen MJ, et al. (2013) Effects of an isocaloric healthy Nordic diet on insulin sensitivity, lipid profile and inflammation markers in metabolic syndrome - a randomized study (SYSDIET). J Intern Med 274, 52-66.

13. Kanerva N, Kaartinen NE, Schwab U, et al. (2013) The Baltic Sea Diet Score: a tool for assessing healthy eating in Nordic countries. Public Health Nutr 4, 1-9.

14. Kanerva N, Kaartinen NE, Schwab U, et al. (2013) Adherence to the Baltic Sea diet consumed in the Nordic countries is associated with lower abdominal obesity. Br J Nutr 109, $520-528$.

15. Heistaro A (2005) Methodology Report: Health 2000 Survey no. B 6/2005. Helsinki: National Public Health Institute.

16. Vartiainen E, Laatikainen T, Peltonen M, et al. (2010) Thirtyfive-year trends in cardiovascular risk factors in Finland. Int J Epidemiol 39, 504-518.

17. Eriksson JG, Forsen T, Tuomilehto J, et al. (2002) Effects of size at birth and childhood growth on the insulin resistance syndrome in elderly individuals. Diabetologia 45, 342-348.

18. Konttinen H, Männistö S, Sarlio-Lähteenkorvä S, et al. (2010) Emotional eating, depressive symptoms and self-reported food consumption. A population-based study. Appetite 54, 473-479.

19. Männistö S, Virtanen M, Mikkonen T, et al. (1996) Reproducibility and validity of a food frequency questionnaire in a case-control study on breast cancer. J Clin Epidemiol 49, 401-409.

20. Kaartinen NE, Tapanainen H, Valsta LM, et al. (2012) Relative validity of a FFQ in measuring carbohydrate fractions, dietary glycaemic index and load: exploring the effects of subject characteristics. Br J Nutr 107, 1367-1375.

21. Paalanen L, Männistö S, Virtanen MJ, et al. (2006) Validity of a food frequency questionnaire varied by age and body mass index. J Clin Epidemiol 59, 994-1001.
22. Reinivuo H, Hirvonen T, Ovaskainen ML, et al. (2010) Dietary survey methodology of FINDIET 2007 with a risk assessment perspective. Public Health Nutr 13, 915-919.

23. Tolonen H, Koponen P, Aromaa A, et al. (2008) Recommendations for the Health Examination Surveys in Europe no. B21/2008. Helsinki: National Public Health Institute.

24. Ylihärsilä H, Kajantie E, Osmond C, et al. (2008) Body mass index during childhood and adult body composition in men and women aged 56-70 y. Am J Clin Nutr 87, 1769-1775.

25. Fossati P \& Prencipe L (1982) Serum triglycerides determined colorimetrically with an enzyme that produces hydrogen peroxide. Clin Chem 28, 2077-2080.

26. Lie RF, Schmitz JM, Pierre KJ, et al. (1976) Cholesterol oxidasebased determination, by continuous-flow analysis, of total and free cholesterol in serum. Clin Chem 22, 1627-1630.

27. Lopes-Virella MF, Stone P, Ellis S, et al. (1977) Cholesterol determination in high-density lipoproteins separated by three different methods. Clin Chem 23, 882-884.

28. Friedewald WT, Levy RI \& Fredrickson DS (1972) Estimation of the concentration of low-density lipoprotein cholesterol in plasma, without use of the preparative ultracentrifuge. Clin Chem 18, 499-502.

29. Alberti KG, Eckel RH, Grundy SM, et al. (2009) Harmonizing the metabolic syndrome: a joint interim statement of the International Diabetes Federation Task Force on Epidemiology and Prevention; National Heart, Lung, and Blood Institute; American Heart Association; World Heart Federation; International Atherosclerosis Society; and International Association for the Study of Obesity. Circulation 120, $1640-1645$.

30. Pearson TA, Mensah GA, Alexander RW, et al. (2003) Markers of inflammation and cardiovascular disease: application to clinical and public health practice: a statement for healthcare professionals from the Centers for Disease Control and Prevention and the American Heart Association. Circulation 107, 499-511.

31. R Development Core Team (2012) R: A Language and Environment for Statistical Computing. Vienna: R Foundation for Statistical Computing. http://www.R-project.org/ (accessed June 2013).

32. Rothman KJ (1986) Modern Epidemiology, 1st ed. Boston, MA: Little, Brown and Company.

33. Black AE (2000) Critical evaluation of energy intake using the Goldberg cut-off for energy intake:basal metabolic rate. A practical guide to its calculation, use and limitations. Int J Obes Relat Metab Disord 24, 1119-1130.

34. Goldberg GR, Black AE, Jebb SA, et al. (1991) Critical evaluation of energy intake data using fundamental principles of energy physiology: 1 . Derivation of cut-off limits to identify under-recording. Eur J Clin Nutr 45, 569-581.

35. World Health Organization (1985) Energy and Protein Requirements. Report of a Joint WHO/FAO/UNU Expert Consultation. WHO Technical Report Series no. 724. Geneva: WHO.

36. Smith-Warner SA, Spiegelman D, Ritz J, et al. (2005) Methods for pooling results of epidemiologic studies. Am J Epidemiol 163, 1053-1064.

37. Torres JL \& Ridker PM (2003) Clinical use of high sensitivity C-reactive protein for the prediction of adverse cardiovascular events. Curr Opin Cardiol 18, 471-478.

38. Calabro P, Golia E \& Yeh ET (2009) CRP and the risk of atherosclerotic events. Semin Immunopathol 31, 79-94.

39. Emerging Risk Factors Collaboration, Kaptoge S, Di Angelantonio E, et al. (2012) C-reactive protein, fibrinogen, and cardiovascular disease prediction. $N$ Engl J Med 367, $1310-1320$. 
40. Lazarou C, Panagiotakos DB, Chrysohoou C, et al. (2010) C-reactive protein levels are associated with adiposity and a high inflammatory foods index in mountainous Cypriot children. Clin Nutr 29, 779-783.

41. North CJ, Venter CS \& Jerling JC (2009) The effects of dietary fibre on C-reactive protein, an inflammation marker predicting cardiovascular disease. Eur J Clin Nutr 63, 921-933

42. de Mello VD, Schwab U, Kolehmainen M, et al. (2011) A diet high in fatty fish, bilberries and wholegrain products improves markers of endothelial function and inflammation in individuals with impaired glucose metabolism in a randomised controlled trial: the Sysdimet study. Diabetologia 54, 2755-2767.

43. Brinton EA (2012) Effects of ethanol intake on lipoproteins Curr Atheroscler Rep 14, 108-114.

44. Aller EE, Abete I, Astrup A, et al. (2011) Starches, sugars and obesity. Nutrients 3, 341-369.

45. Sacks FM \& Katan M (2002) Randomized clinical trials on the effects of dietary fat and carbohydrate on plasma lipoproteins and cardiovascular disease. Am J Med 113, Suppl. 9B, 13S-24S.

46. Siri-Tarino PW, Sun Q, Hu FB, et al. (2010) Saturated fat, carbohydrate, and cardiovascular disease. Am J Clin Nutr 91, 502-509.

47. Hooper L, Abdelhamid A, Moore HJ, et al. (2012) Effect of reducing total fat intake on body weight: systematic review and meta-analysis of randomised controlled trials and cohort studies. BMJ 345, e7666.

48. Nordic Council of Ministers (2004) Nordic Nutrition Recommendations 2004, 4th ed. Copenhagen: Nord.
49. Working Group of Finnish Medical Society Duodecim and Finnish Society of Internal Medicine (2013) Current Care Guidelines for Dyslipidaemias: Summary. http://www. kaypahoito.fi/web/english/summaries/naytaartikkeli/tunnus/ ccs00055 (accessed December 2012).

50. Willett WC (2013) Food frequency methods. In Nutritional Epidemiology, 3rd ed., p. 70. New York, NY: Oxford University Press.

51. Benitez-Arciniega AA, Mendez MA, Baena-Diez JM, et al. (2011) Concurrent and construct validity of Mediterranean diet scores as assessed by an FFQ. Public Health Nutr 14, 2015-2021.

52. Livingstone MB \& Black AE (2003) Markers of the validity of reported energy intake. J Nutr 133, Suppl. 3, 895S-920S

53. Leiviskä J, Sundvall J, Alfthan G, et al. (2013) What have we learnt about high-density lipoprotein cholesterol measurements during 32 years? Experiences in Finland 1980-2012. Clin Chim Acta 415, 118-123.

54. Superko HR, Pendyala L, Williams PT, et al. (2012) High-density lipoprotein subclasses and their relationship to cardiovascular disease. J Clin Lipidol 6, 496-523.

55. Williams PT \& Feldman DE (2011) Prospective study of coronary heart disease vs. HDL2, HDL3, and other lipoproteins in Gofman's Livermore Cohort. Atherosclerosis 214, 196-202.

56. Voight BF, Peloso GM, Orho-Melander M, et al. (2012) Plasma HDL cholesterol and risk of myocardial infarction: a mendelian randomisation study. Lancet 380, 572-580. 